\title{
The SoftPro project: Synergy-based Open-source Technologies for Prosthetics and Rehabilitation
}

\author{
Cristina Piazza, Manuel G. Catalano, Matteo Bianchi, Emiliano Ricciardi, Domenico Prattichizzo, Sami Haddadin, \\ Andreas R. Luft, Olivier Lambercy, Roger Gassert, Eike Jakubowitz, Herman Van Der Kooij, Frederick Tonis, \\ Fabio Bonomo, Benjamin de Jonge, Tomas Ward, Kristin D. Zhao, Marco Santello, and Antonio Bicchi
}

\begin{abstract}
Robotics-enabled technologies for assistive and rehabilitative applications have gained an increasing attention, both in academic and industrial research settings, as a promising solution for human sensory-motor system recovery. However, many constraints remain that limit their effective employment in everyday-life, mainly related to cost, usability and users' acceptance. The Softpro project proposes to completely reverse such paradigm, starting from the analysis of the needs from patients and the careful investigation of the sensory-motor human behaviour, capitalizing on the framework of synergistic control and soft robotics. The final goal is to study and design simple, effective and affordable soft synergy-based robotic technologies for the upper limb, such as new prostheses, exoskeletons, and assistive devices which can be useful and accessible to a wide audience of users. To pursue such an ambitious objective, SoftPro has put together research groups who laid the neuroscientific and technological fundamentals underpinning the project approach, a net of international collaborations and numerous and qualified industrial partners, which is expected to produce a strong impact on both research and innovation.
\end{abstract}

\section{INTRODUCTION}

CURRENTLY availabe technolgy-enabled rehab training and assistive devices are very rarely manageable, intuitive, and affordable enough to be used in patients' everyday life. The ambitious objective of the SoftPro project [1] firmly adheres to a quantitative assessment-based philosophy in the development of innovation concerning prosthetics

This research has received funding from the European Union's Horizon 2020 Research and Innovation Programme under Grant Agreement No.688857 (SoftPro). The content of this publication is the sole responsibility of the authors. The European Commission or its services cannot be held responsible for any use that may be made of the information it contains.

C. P., M. B., A. B. are with University of Pisa, Pisa, Italy (corresponding author: cristina.piazza@ing.unipi.it).

M.G. C., D. P., A. B. are with Istituto Italiano di Tecnologia, Genoa, Italy

E. R. is with IMT School for Advanced Studies Lucca, Lucca, Italy

D. P. is with University of Siena, Siena, Italy

S. H. is with Technische Universitat Munchen, Munchen, Germany

A. R. L. is with University Hospital Zurich, Zurich, Switzerland

R. G., O. L. are with ETH Zurich, Zurich, Switzerland

E. J. is with Hannover Medical School, Hannover, Germany

H. V. is with University of Twente, Enschede, Netherlands

F. T. is with Hankamp Rehab, Enschede, Netherlands

F. B. is with qbrobotics, Navacchio, Italy

B. J. is with TMS International, Enschede, Netherlands

T. W. is with Bioservo Technologies AB, Kista, Sweden

K. Z. is with Mayo Clinic, Rochester (MN), Usa

M. S. is with Arizona State University, Tempe, Arizona

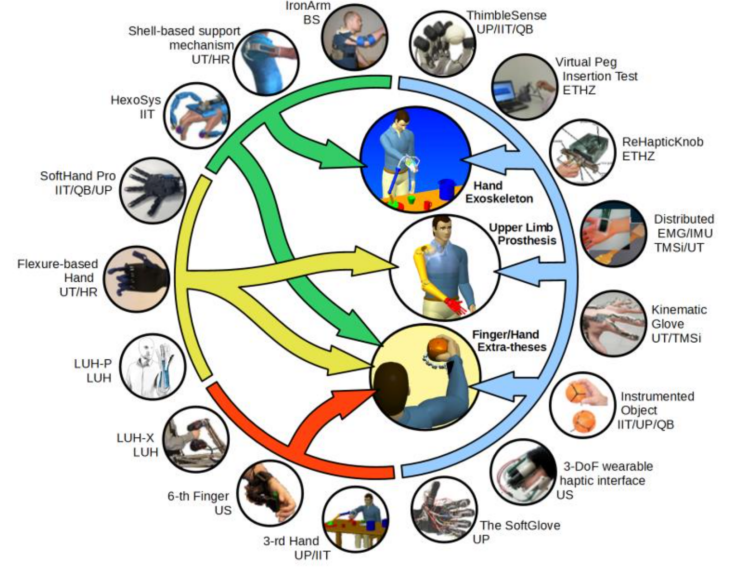

Fig. 1. SoftPro technologies and integration

and neurorehabilitation devices. Starting from homogeneous methodological bases, the aim of the project is to address a spectrum of challenges ranging from technologies that, starting from a validation in relevant environments, reach a complete and qualified status, to high-risk high-gain ideas supported only by preliminary observations that will hopefully reach a relevant validation stage. Three aspects are crucial in our project: the control of prostheses and rehab devices, where the human "talks" to the artificial device to control its motion through brain- and body-machine interfaces; the haptic stimulus delivery, where the artificial "talks" to the human to render the missing or deficient sensory feedback; and the implementation of elementary semi-autonomous sensorimotor loops, such as the grip reflex in a hand prosthesis or exoskeleton.

\section{MATERIALS AND METHODS}

Based on the previous work of its research and industrial participants and in close collaboration with clinical participants, The SoftPro project is working on the development of new advanced instruments to measure the dynamics of interaction between the user, the assistive device and the environment (other people and objects in the surroundings) in new and more accurate ways. In SoftPro, starting from a solid neuroscientific theoretical basis on sensorimotor synergies as the elementary alphabet of human motor control primitives 
and from the theory and technology of soft robotics that enable controllable impedance and adaptability in physical human-robot interaction, new methods and technologies for prosthetics and rehabilitation can be developed. Furthermore, a principled simplification approach allows the development of the simplest technology to fulfill a desired assistive goal. This approach is then extended to the design of upper limb and hand prostheses with a larger/different set of synergies, and supernumerary limbs for assistance and rehabilitation (extratheses) to increase upper limb functionality and subsequent independence in activities of daily living (ADLs) in persons where no more functional motor improvements seem to be achievable. The SoftPro principled simplification approach also provides for hand and upper limb exoskeletons that are light-weight, low-cost, easy to don and only minimally interfere with natural motion and interaction with the environment. Finally, to maximize the impact of our research we are following a very open approach to sharing our results. SoftPro promotes open access not only to data collected, but also to technology developed, for the purpose of building a community of users and developers which will in turn contribute to furthering our goals of making prosthetics and rehabilitation aids more easily accessible.

\section{RESULTS}

Main results currently achieved in applied neuroscience include the clarification of the theory of sensorimotor synergies in rehabilitation ([2]), and the development of novel algorithms for eliciting synergy-inspired control of robotic aids from brain-machine interfaces. The SoftPro project is further pursuing the concept of soft-synergy based hand prostheses (the SoftHand Pro, [3]), currently being tested at a wide range of international facilities, at a pre-marketable state (TRL8). New tools for the assessment of effectiveness of robot-enabled prostheses and assistive devices include the maturation of the Virtual Peg Insertion Test, the development and application of force/torque based [4], intrinsic tactile sensing techniques to measure grasping and manipulative forces with tools such as instrumented objects and the ThimbleSense [5]. Moreover, other SoftPro devices are currently reaching a higher TRL, as e.g. the shell-based exoskeletons, and IMU-based posture and measurement gloves [6]. Starting from previous works by participans, as the HexoSys [7] or the HandExo [8], the SoftPro exoskeletons will be designed to achieve a reliable construction with more optimized kinematics, using suitable materials, small form factor, low weight and high precision. The design and validation of novel interfaces for haptic stimulus delivery in prosthetics and robotics rehab, represents a significant improvement for closing the sensory-motor loop in an intuitive and simple manner, likely contributing to device acceptance, as e.g. [9], [10]. Finally, SoftPro is featuring "red label" investigations and developments, where the project is undertaking higher risks testing disruptively new hypotheses and paradigms. These include the attempt to estimate from autonomic nervous system signals, subtle but important information such as stress and/or fatigue [11]; and pioneering the idea of using

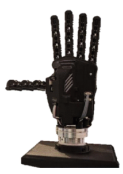

(a)

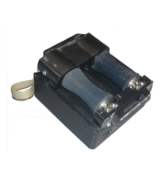

(b)

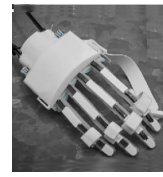

(c)

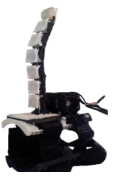

(d)
Fig. 2. Examples of devices developed or used within the SoftPro project: (a) SoftHand Pro, (b) Stretch Pro, (c) HandExo and (d) Sixth Finger.

robotic extra limbs [12] for assistance to persons with chronic motor impairments.

\section{CONCLUSION}

The SoftPro project addresses the scope to advance key technologies for assistive and rehabilitative robotics. In terms of innovation, SoftPro capitalizes on previous research work by the participants, often in collaboration, to significantly progress in engineering, clinical validation, and assess the economic viability of systems for robotics-enabled aids. Thanks to the strong links between participants, the industrial pickup of at least some of these results is highly probable.

\section{REFERENCES}

[1] SoftPro Project website http://www.softpro.eu/

[2] Leo A., Handjaras G., Bianchi M., Marino H., Gabiccini M. Guidi A. Scilingo E. P., Pietrini P., Bicchi A., Santello M., Ricciardi E. (2016). A synergy-based hand control is encoded in human motor cortical areas. Elife, vol. 5, eLife Sciences Publications, Ltd.

[3] Piazza C., Catalano M. G., Godfrey S. B., Rossi M., Grioli G., Bianchi M., Zhao K., Bicchi A. (2017). The SoftHand Pro-H: A Hybrid BodyControlled, Electrically Powered Hand Prosthesis for Daily Living and Working. IEEE Robotics \& Automation Magazine, vol. 24(4), 87-101

[4] Hofmann P., Held J. P., Gassert R., Lambercy O. (2016, July). Assessment of movement patterns in stroke patients: a case study with the Virtual Peg Insertion Test. In Proceedings of the international Convention on Rehabilitation Engineering \& Assistive Technology ( $\mathrm{p}$. 14). Singapore Therapeutic, Assistive \& Rehabilitative Technologies (START) Centre

[5] Battaglia E., Grioli G., Catalano M. G., Santello M., Bicchi A. (2014, May). ThimbleSense: an individual-digit wearable tactile sensor for experimental grasp studies. In Robotics and Automation (ICRA), 2014 IEEE International Conference on (pp. 2728-2735). IEEE.

[6] Santaera, G., Luberto, E., Serio, A., Gabiccini, M., Bicchi, A. (2015, May). Low-cost, fast and accurate reconstruction of robotic and human postures via IMU measurements. In Robotics and Automation (ICRA), 2015 IEEE International Conference on (pp. 2728-2735). IEEE.

[7] Iqbal, J., Tsagarakis, N. G., Caldwell, D. G. (2011, August). A multiDOF robotic exoskeleton interface for hand motion assistance. In Engineering in Medicine and Biology Society, EMBC, 2011 Annual International Conference of the IEEE (pp. 1575-1578). IEEE.

[8] Nycz, C. J., Bützer, T., Lambercy, O., Arata, J., Fischer, G. S., Gassert, R. (2016). Design and characterization of a lightweight and fully portable remote actuation system for use with a hand exoskeleton. IEEE Robotics and Automation Letters, 1(2), 976-983.

[9] Rossi M., Bianchi M., Battaglia E., Catalano M. G., Bicchi A. (2018) Hap-Pro: a wearable haptic device for proprioceptive feedback. IEEE Transactions on Biomedical Engineering

[10] Meli L., Hussain I., Aurilio M., Malvezzi M., O’Malley M., Prattichizzo D. (2018). The hBracelet: a wearable haptic device for the distributed mechanotactile stimulation of the upper limb. IEEE Robotics and Automation Letters.

[11] Peternel, L., Tsagarakis, N., Caldwell, D., Ajoudani, A. (2017). Robot adaptation to human physical fatigue in human-robot co-manipulation. Autonomous Robots, 1-11.

[12] G. Salvietti, I. Hussain, D. Prattichizzo. The Robotic Sixth Finger: A Wearable Compensatory Tool to Regain Grasping Capabilities in Paretic Hands. In Robotics Research, Vol. 2, pp. 423-437, Springer, 2018. 